\title{
Special Issue: First International Conference on Mechanical Engineering (INCOM-2018)
}

\section{Foreword}

Published online 31 August 2020

This special issue contains selected papers from among those presented at the "First International Conference on Mechanical Engineering (INCOM)', 2018 held from 4 to 6 January, 2018 at Jadavpur University, Kolkata. The conference was organized by the Department of Mechanical Engineering, Jadavpur University. Around 200 papers were presented at the conference in wide ranging areas of mechanical engineering.

Based on a preliminary scrutiny, some papers, mostly based on fundamental areas of mechanical engineering, were shortlisted for consideration of publication in the special issue of Sadhana. The authors of the shortlisted papers were invited to submit extended versions of their works presented at the conference. The submitted papers were reviewed following the review protocol of Sadhana. After multiple rounds of review, eleven papers were finally selected. These papers, apart from being of high quality that meets the standards of the journal, represent different areas like solid mechanics and materials, fluid-structure interaction, heat transfer, combustion and dynamic analysis of mechanical systems.

We are grateful to all the reviewers for their sustained cooperation. We also thank the Chief Editors and staff of Sadhana journal for their enthusiastic help. We hope that the range of papers and their contents will be of interest to the readers.

July 2020

ACHINTYA MUKHOPADHYAY

Jadavpur University, Kolkata, India

e-mail: achintya.mukho@gmail.com
SUMIT BASU

Indian Institute of Technology, Kanpur, India e-mail:sbasu@iitk.ac.in

(Guest Editors)

This paper is a revised and expanded version of an article presented in "First International Conference on Mechanical Engineering" held at 'Jadavpur University', Kolkata, India during January 4-6, 2018 (INCOM-2018). 